\title{
Sleep characteristics of individuals with chronic stroke: a pilot study
}

\author{
This article was published in the following Dove Press journal: \\ Nature and Science of Sleep \\ 21 October 2015 \\ Number of times this article has been viewed
}

\author{
Mayis Al-Dughmi' \\ Alham Al-Sharman ${ }^{2}$ \\ Suzanne Stevens ${ }^{3}$ \\ Catherine F Siengsukon' \\ 'Department of Physical Therapy and \\ Rehabilitation Science, University \\ of Kansas Medical Center, Kansas \\ City, KS, USA; ${ }^{2}$ Department of \\ Rehabilitation Sciences, Jordan \\ University of Science and Technology, \\ Irbid, Jordan; ${ }^{3}$ Department of \\ Neurology, University of Kansas \\ Medical Center, Kansas City, KS, USA
}

Correspondence: Catherine F Siengsukon Department of Physical Therapy and Rehabilitation Science, University of Kansas Medical Center, 390I Rainbow Boulevard, Mail Stop 2002, Kansas City, KS 66160, USA

$\mathrm{Tel}+19135886913$

Fax + 9135884568

Email csiengsukon@kumc.edu

\begin{abstract}
Changes in sleep characteristics in individuals with chronic stroke are not well described, particularly compared with healthy individuals. Therefore, the aim of this pilot study was to explore the sleep characteristics in individuals with chronic stroke compared to age- and sex-matched controls. Sixteen individuals with chronic stroke and ten age- and sex-matched controls underwent two nights of polysomnographic recording. The sleep characteristics of interest included total sleep time, sleep efficiency, and percent time, as well as time in minutes spent in stages N1, N2, and N3 and stage R sleep. The individuals with chronic stroke spent less percent time in stage $\mathrm{N} 3$ compared with controls $(P=0.048)$. No significant differences in the other sleep characteristics were found between the stroke and control groups. Individuals with chronic stroke present with altered stage N3 sleep compared with healthy controls. These alterations in stage N3 sleep might be a sign of neuronal dysfunction and may impact recovery following stroke. A larger scale study is needed to confirm these findings.
\end{abstract}

Keywords: sleep characteristics, sleep, chronic stroke, polysomnography

\section{Introduction}

Individuals after stroke often experience sleep disturbances and changes in sleep patterns. Up to $40 \%$ of individuals with chronic stroke (defined as $>6$ months following stroke) and $70 \%$ of those with acute stroke have sleep-wake disorders, including excessive daytime sleepiness, insomnia, hypersomnia, and fatigue. ${ }^{1}$ Sleep-related breathing disturbances are also common, affecting up to $70 \%$ of individuals with stroke, ${ }^{2}$ with obstructive sleep apnea being the most common form. ${ }^{2}$ Sleep-related movement disorders like restless leg syndrome and periodic limb movements, as well as stage $\mathrm{R}$ (formally referred to as rapid eye movement [REM]) sleep behavior disorder are also common in individuals with stroke. ${ }^{3}$ Sleep disturbances following stroke can lead to short-term and long-term negative patient outcomes ${ }^{4}$ and increase the risk of a recurrent stroke..$^{5}$ Medication use, sleep-disordered breathing, depression, pain, impaired mobility, and other complications from the stroke are factors that contribute to compromised sleep in people following stroke. ${ }^{6}$ Furthermore, the area of the brain affected by the stroke can play an important role in determining the spectrum of sleep disturbances that occur. ${ }^{6}$ In addition, untreated sleep disturbances after stroke, like sleep apnea and excessive daytime sleepiness, increase the risk of developing another stroke.?

Sleep has been shown to enhance learning motor skills and memory consolidation in individuals with chronic stroke. ${ }^{8}$ 
Sleep is classified into two main stages: ${ }^{9}$ non-REM sleep (stage N) or REM (stage R) sleep. Stage N sleep is divided into three substages corresponding to increasing depth of sleep as shown using electroencephalograms (EEGs): N1 involves the transition from wakefulness to sleep and is characterized by sinusoidal alpha wave activity, N2 is characterized by sleep spindles and K complexes (clusters of low- and high-amplitude waves, respectively), and N3 or slow-wave sleep is characterized by slow delta waves. Stage R sleep is characterized by REMs and muscle atonia.

Evidence suggests that sleep spindles and possibly stage R sleep are associated with consolidation of simple motor tasks during sleep in healthy individuals. ${ }^{10}$ Therefore, understanding the sleep characteristics in individuals with stroke could enable clinicians to capitalize on sleep to promote learning and hasten recovery. Furthermore, it would enable clinicians to treat sleep issues that may limit the individual's ability to benefit from sleep to enhance motor learning and recovery.

While sleep disturbances and disorders in individuals with stroke can lead to an additional stroke and may impact the ability to learn new motor skills, ${ }^{7}$ sleep issues also negatively impact functional outcomes and overall quality of life in individuals with stroke. ${ }^{11}$ Furthermore, there is evidence that poor sleep quality and sleep disruptions compromise the immune system, ${ }^{12}$ alter the tissue healing process, ${ }^{13}$ increase pain sensitivity, ${ }^{14}$ contribute to depression and anxiety, ${ }^{15}$ and reduce quality of life and functional well-being. ${ }^{16}$ All of these comorbid conditions are commonly experienced by people following stroke, although the contribution of sleep to these comorbid conditions has not been fully examined in people with stroke. Therefore, exploring the sleep characteristics in individuals with stroke could lead to a better understanding of the contribution of sleep to the development of comorbid conditions in people with stroke.

Previous studies ${ }^{11,17-27}$ that used polysomnography (PSG) to examine sleep characteristics in individuals with stroke showed sleep alterations as following: decreased sleep efficiency (SE), reduced total sleep time (TST), increased time spent in stage N1 sleep, and decreased percentage of time spent in stage N3 or slow-wave sleep. ${ }^{12,18-24,26-29}$ There is also evidence that sleep spindles increase in activity in the chronic stage of stroke, ${ }^{21,28}$ as well as increased time spent in stage $\mathrm{N} 2$ in the chronic phase.$^{30}$ However, these studies have several limitations in their design. First, only four of the 12 PSG studies included healthy participants as their control group $;^{19,22,23}$ the remaining used hospitalized patients ${ }^{17,18,25}$ or other patients with neurologic disease. ${ }^{20,21,27}$ Neurologically intact, sex- and aged-matched controls would be an ideal comparison to reduce the impact of medications or comorbidities that might impact sleep characteristics, thus making conclusions difficult. In addition, most of the PSG studies focus on individuals with acute stroke ${ }^{11,20,21,25}$ or included individuals with a diverse range of chronicity ranging from acute, subacute, to chronic stages. ${ }^{18,22}$ Therefore, evidence of sleep characteristics in individuals with chronic stroke of greater than 6 months is lacking. Understanding the sleep characteristics of individuals with chronic stroke is especially critical because approximately 6.8 million Americans are living with chronic stroke, ${ }^{29}$ and up to $50 \%$ of those with chronic stroke report having sleep disturbances. ${ }^{31}$ Another difficulty in interpreting the findings of prior studies is the majority were conducted on a single night. ${ }^{18-20,22-24,26-28,32}$ Two nights of PSG recording is recommended, where the first night is referred to as the "adaptation" or "acclimation" night and is disregarded from analysis and considered not representative of the usual sleeping patterns. ${ }^{33}$ Only two studies conducted PSG on two to three consecutive nights, but their target population was individuals with acute stroke. ${ }^{19,27}$ To our knowledge, there are no studies that have assessed the sleep characteristics of individuals with chronic stroke using a healthy sex- and age-matched control group for comparison and using an acclimation night.

Therefore, the purpose of this pilot study was to analyze the sleep characteristics of individuals with chronic stroke (defined as more than 6 months poststroke) compared with healthy age- and sex-matched controls using PSG conducted on two nights (first night was used for acclimation). A secondary purpose was to make an exploratory analysis to compare the sleep characteristics of those with right-sided with those with left-sided middle cerebral artery (MCA) lesions.

\section{Methods}

The study was conducted according to the regulations and with approval from the Institutional Review Board at the University of Kansas Medical Center (KUMC). Informed consent was received from all participants. Participants were recruited from 1) the Stroke Registry at KUMC, 2) the American Stroke Foundation, 3) area stroke support groups, and 4) personal referral from consented subjects, area physicians, or study personnel. The inclusion criteria for all participants were as follows: 1) 40-75 years old, 2) no untreated sleep disorders including sleep apnea or narcolepsy, 3) must maintain a regular sleep schedule (defined 
for the purpose of this study as an average of 6-9 hours of sleep per night), and 4) score at least 26 on the Mini-Mental State Exam to insure no evidence of dementia. The inclusion criteria for participants with stroke were 1) unilateral stroke in the MCA distribution, and 2) current clinical status being at least 6 months poststroke. The exclusion criteria for all participants were as follows: 1) acute medical problems, 2) uncorrected vision loss, 3) previous history of psychiatric admission, 4) history of multiple strokes, transient ischemic attacks, or extensive white matter disease, 5) unable to give consent, 6) smoker, and 7) uncontrolled depression.

Twenty-six individuals with stroke were enrolled in the study. Six individuals were excluded from the study after the night of PSG due to having undiagnosed sleep apnea. Ten ageand sex-matched controls were included. Control participants were matched to two stroke participants for 1:2 ratio. Three individuals with stroke were identified as extreme outliers on box-plot test for normality of the sleep measures and were excluded from data analysis. One stroke participant was excluded because magnetic resonance imaging revealed a brainstem lesion. Therefore, 16 individuals with chronic stroke (mean age $=57 \pm 10.34$ years; eleven females and five males; Table 1) and ten sex- and age-matched control participants (mean age $=60.8 \pm 11.68$ years; six females and four males; Table 1) were included in data analysis.

Participants underwent a night of acclimation sleep in the Sleep Medicine Clinic sleep laboratory at the University of Kansas Medical Center to acclimate individuals to the sleep laboratory and to exclude participants with a sleep disorder. The acclimation night was followed by a night for sleep data recording. Only the second night of recording was included in data analysis. The participants underwent a third night of PSG to examine the sleep parameters associated with sleepdependent off-line motor skill learning, and those data are not presented here.

Participants were prepared for polysomnographic sleep monitoring using standardized techniques ${ }^{34}$ including six EEG sensors to measure brain wave activity placed according to the international system for electrode placement, ${ }^{35}$ two eye sensors to record horizontal eye movement (electro- oculogram), and chin and tibialis anterior electromyogram sensors to assess muscle activity. Physiological signals were recorded using Nihon Kohden equipment (Foothill Ranch, CA, USA). Other instruments included a nasal airflow sensor, comfortable belts around the thorax and abdomen to monitor respiratory effort, a finger probe to assess pulse oximetry, and a microphone to monitor snoring. Each study session was digitally video recorded. The sleep records were scored according to standardized scoring criteria. ${ }^{36}$ The outcome measures of interest are as follows: TST, SE, time spent in stage N1, stage N2, stage N3, and stage R, and percent sleep period time spent in stage N1, stage N2, stage N3, and stage R. For data analysis, SPSS version 20.0 was used to perform all statistical analysis, and alpha was set at 0.05 . Descriptive statistics were calculated. The data were analyzed for normality using the Shapiro-Wilk test and Skewness and Kurtosis test, as well as Box plots and normal Q-Q plots. Independent $t$-tests were used to compare the sleep parameters of the individuals with stroke with the control participants when the assumption of normality was satisfied. Effect sizes were calculated as Cohen's $d$ values. Because this is a pilot study, data are presented without correction for multiplicity. Due to the sex difference in the stroke sample, linear regression was used to study if sex predicts the outcome measure of interest after controlling for age which can be a confounding factor for change in sleep parameters. An exploratory analysis was performed using independent $t$-tests to compare the sleep characteristics and descriptive characteristics of individuals with left-sided MCA lesions $(n=7)$ to those with right-sided MCA lesions $(n=9)$. Alpha was set at 0.05 .

\section{Results}

There was no significant difference in age $(P=0.504)$, MiniMental State Exam $(P=0.924)$, Pittsburgh Sleep Quality Index $(P=0.455)$, Geriatric Depression Scale $(P=0.840)$, or average sleep for the week prior to testing $(P=0.062)$ between the stroke participants and the healthy controls (Table 1). The participants with stroke showed higher TST than the control participants (414.75 minutes vs 388.3 minutes), but this was not statistically different ( $P=0.058$; Table 2$)$.

Table I Demographics of the participants

\begin{tabular}{lllllllll}
\hline Group & Sex & Age (years) & MMSE & PSQI & GDS & AVG sleep & UEFM & OPS \\
\hline Stroke & II females 5 males & $57(10.34)$ & $29.43(0.73)$ & $4.5(3.85)$ & $5.36(4.70)$ & $6.56(2.52)$ & $56.88($ I4.23) & I.85 (0.4I) \\
Control & 6 females 4 males & $60.8($ II.68) & $30(0)$ & $4(2.3 I)$ & $3.2(4.23)$ & $7.467(0.657)$ & N/A & N/A \\
P-value & & 0.504 & 0.924 & 0.455 & 0.840 & 0.062 & & \\
\hline
\end{tabular}

Note: Data are reported as mean (standard deviation).

Abbreviations: MMSE, Mini-Mental State Exam; PSQI, Pittsburgh Sleep Quality Index; GDS, Geriatric Depression Scale; AVG sleep, average hours of sleep over I week; UEFM, upper extremity portion of the Fugl-Meyer Assessment of Motor Recovery After Stroke; OPS, Orpington Prognostic Score. 
Table 2 Descriptive mean statistics of sleep parameters in the stroke and control participants

\begin{tabular}{|c|c|c|c|c|c|}
\hline Variable & Stroke & Control & $\begin{array}{l}\text { Shapiro-Wilk } \\
\text { test ( } P \text {-values) }\end{array}$ & Cohen's d & $\begin{array}{l}t \text {-test unless noted } \\
\text { as } U \text { test ( } P \text {-values) }\end{array}$ \\
\hline TRT (min) & $469.06(24.7 I)$ & $465.50(22.97)$ & $0.930(0.242)$ & 0.149 & $0.367(0.901)$ \\
\hline TST (min) & 414.75 (33.69) & $388.30(57.55)$ & $0.967(0.790)$ & 0.560 & $\mathrm{I} .485(0.058)$ \\
\hline Stage NI (min) & $33.56(12.57)$ & $41.20(15.52)$ & $0.834(0.008)$ & -0.540 & $U=55.5(0.20 I)$ \\
\hline Stage N2 (min) & $256.44(38.92)$ & 215.40 (57.98) & $0.923(0.190)$ & 0.831 & $2.167(0.270)$ \\
\hline Stage N3 (min) & $43.06(26.84)$ & $46.80(33.87)$ & $0.949(0.476)$ & -0.122 & $-0.312(0.216)$ \\
\hline Stage R (min) & $81.69(27.04)$ & $88.9(30.16)$ & $0.837(0.009)$ & -0.252 & $U=71.5(0.660)$ \\
\hline Stage NI (\%) & $8.32(3.09)$ & $9.5(4.37)$ & $0.857(0.018)$ & -0.312 & $U=6 \mathrm{I}(0.336)$ \\
\hline Stage N2 (\%) & $61.74(8.27)$ & $54.8(8.62)$ & $0.958(0.622)$ & 0.823 & $2.05(0.805)$ \\
\hline Stage N3 (\%) & $9.86(5.94)$ & $12.6(|0.4|)$ & $0.933(0.27 I)$ & -0.323 & $-0.860(0.048)$ \\
\hline Stage R (\%) & $19.3(6.15)$ & $20.3(9.08)$ & $0.799(0.003)$ & -0.129 & $U=76(0.856)$ \\
\hline Sleep efficiency (\%) & $88.64(6.79)$ & $83.5(11.46)$ & $0.856(0.017)$ & 0.546 & $U=49.5(0.109)$ \\
\hline
\end{tabular}

Notes: Data are reported as mean (standard deviation), Shapiro-Wilk test statistic ( $P$-values), values of Cohen's $d$, and $t$-test statistic or Mann-Whitney $U$ test $(P$-values).

Abbreviations: TRT, total recording time; TST, total sleep time; min, minutes.

The individuals with stroke had higher SE than the control participants, but this difference was not significant $(88.64 \%$ vs $83.5 \%, P=0.109$; Table 2). The stroke group also spent less time than controls in the following stages of sleep: stage N1 (33.56 minutes vs 41.2 minutes, $P=0.201$ ), stage N3 (43.06 minutes vs 46.8 minutes, $P=0.216$ ), and stage $\mathrm{R}$ ( 81.69 minutes vs 88.9 minutes, $P=0.660$ ) but more time in stage N2 compared with controls (256.44 minutes vs 215.4 minutes, $P=0.270$; Table 2), although not statistically significant. When comparing the percentage of time spent in each stage, the individuals with stroke had a significant lower percent time spent in stage $\mathrm{N} 3$ compared with controls $(P=0.048)$, but there was no statistically significant difference between the other percent times. The analysis was repeated again for the percent of time spent in stage N3 variable, but this time with all the 19 stroke individuals without taking out the outliers. The analysis showed that there is a near significant difference in the percentage of time spent in stage $\mathrm{N} 3$ between the stroke participants and controls $(P=0.082)$. To show that the percentage of time spent in stage N3 was not influenced by the presence of more females than males in our 16 stroke samples, linear regression was used with stage N3 as the dependent variable and sex as a predictor variable. The overall linear regression model to assess the impact of sex on the percentage of time spent in stage N3 while controlling for age was not significant $(P=0.97)$ with an $R^{2}$ of 0.005 . In addition, age was not significantly different between the female stroke participants compared with the male participants $(P=0.308)$.

Descriptive characteristics for the participants with right-sided $(n=9)$ and left-sided $(n=7)$ stroke are shown in Table 3 . Those with right-sided lesions spent significantly less minutes in stage N3 $(P=0.029)$ and significantly less percent time spent in stage $\mathrm{N} 3(P=0.019)$ compared with those with left-sided lesions. Those with right-sided lesions scored significantly higher on the Orpington Prognostic Score $(P=0.038)$ compared with those with left-sided lesions, indicating individuals with right-sided lesions had higher stroke severity, although both groups would be considered to have mild strokes. ${ }^{37}$ There was no significant difference in other sleep variables between individuals with right- versus left-sided lesions.

\section{Discussion}

To our knowledge, this is the first study that compared sleep parameters of individuals with chronic MCA stroke to age- and sex-matched healthy controls using PSG. Overall, the participants with chronic MCA stroke spent significantly less percent time in stage N3 compared with healthy controls. Also, individuals with chronic

Table 3 Descriptive statistics of the right- $(n=9)$ and left- $(n=7)$ side stroke participants

\begin{tabular}{lllllll}
\hline Group & Age (years) & PSQI & GDS & AVG sleep & UEFM & OPS \\
\hline Right & $53.8(10.76)$ & $5.1 I(5.0 I)$ & $4.67(5.7)$ & $7.8 I(1.06)$ & $56.3(15.39)$ & $1.9(0.52)$ \\
Left & $61.1(8.8)$ & $3.7 I(1.49)$ & $3.86(3.63)$ & $7.33(0.69)$ & $57.6(15.76)$ & $1.71(0.19)$ \\
P-values & 0.507 & 0.103 & 0.674 & 0.661 & 0.892 & 0.038 \\
\hline
\end{tabular}

Note: Data are reported as mean (standard deviation).

Abbreviations: PSQI, Pittsburgh Sleep Quality Index; GDS, Geriatric Depression Scale; AVG sleep, average hours of sleep over I week; UEFM, Upper Extremity portion of the Fugl-Meyer Assessment of Motor Recovery After Stroke; OPS, Orpington Prognostic Score. 
MCA stroke spent less time in stage N1 and stage $\mathrm{R}$, while spending more time in stage N2 compared with the control participants, although these differences were not statistically different.

While this is the first study that has examined the sleep characteristics of individuals with chronic stroke, the results of this study mirror the results of the study of individuals with acute stroke, in which it was found that individuals with acute stroke spend less time in stage N3 sleep. ${ }^{20,24,38}$ A recent study ${ }^{39}$ that investigated changes in the topographic sleep of EEG in acute and chronic (defined as 3 months for their study) stroke participants suggests that higher slow-wave activity during sleep reflects cortical plasticity and could be used as a predictive measure of recovery of patients with stroke. Furthermore, it has been found that higher slow-wave activity during sleep is associated with improved performance on a motor learning task in healthy individuals. ${ }^{40}$ Huber et $\mathrm{a}^{41}$ also suggest that a decrease in slow-wave activity during sleep might be due to inactivity of the extremity and the resultant lack of input to the contralateral cortex. The latter might explain in part the low percentage of time spent in stage N3 in the stroke sample of the current study.

Based on evidence from the literature, ${ }^{39,40}$ the decrease in N3 of chronic stroke participants in the current study may represent neuronal dysfunction. This finding is clinically important as altered changes in neuroplasticity and in normal sleep homeostasis may negatively affect functional recovery and motor learning of individuals with chronic stroke. To explore the association between N3 and functional abilities, a post hoc analysis was done using Pearson's correlations. However, no significant relationship was found between time spent in stage N3 and the Upper Extremity Fugl-Meyer $(r=0.001, P=0.998)$ or the Orpington Prognostic Score $(r=0.143, P=0.599)$. The low sample size of the current study might explain the lack of association between function and stage N3 in the stroke participants. In addition, the Cohen's $d$ value showed a small effect size of percent of time in stage N3 $(-0.3)$, which might be influenced by the low sample size as well. Future studies should investigate these findings and explore more thoroughly if the reduction in time spent in stage N3 has negative functional outcomes in individuals with chronic stroke.

The exploratory data analysis showed participants with a right-side MCA lesion spent significantly less time in stage $\mathrm{N} 3$ reported in minutes and less percentage compared with those with a lesion on the left-side MCA. In addition, those with right-sided lesions had significantly higher stroke severity than those with left-sided lesions. Evidence from previous research ${ }^{39}$ found a decrease in slow-wave sleep in individuals with stroke correlated with higher stroke severity. However, it should be noted that the individuals in the current study would be considered to have mild strokes, ${ }^{37}$ so it would be unlikely that the decrease in time spent in N3 would be due to stroke severity. Furthermore, because exact lesion location is unknown (ie, cortical vs subcortical), it is difficult to interpret this finding. If other studies confirm these results that the difference in stage N3 sleep is due to the location of a lesion, this information could guide clinicians and sleep specialists to more accurately predict the possible sleep quality disturbances in relation to lesion side in individuals with stroke. Future studies should further investigate this finding in individuals with chronic stroke and examine anatomical lesion location in more detail.

When the data from this study were compared with published norms, ${ }^{42}$ the stroke participants had a similar SE (88.64\%) compared with published norms for similar mean age $(87 \%)$, whereas the control participants had a lower SE $(83.5 \%)$. Furthermore, the percentage of time spent stage N1 sleep for stroke (8.3\%) and control participants $(9.5 \%)$ was well below the normative values (around 20\%). ${ }^{42}$ The high SE for the participants in this study and the low percent time spent in stage N1 sleep suggest that the participants in this study overall experience quality sleep. This is further supported by the average Pittsburgh Sleep Quality Index for both the stroke and control participants having a global score below 5 , which indicates good sleep quality. ${ }^{43}$ Because individuals were excluded if an untreated sleep disorder was identified during the acclimation night and if they have uncontrolled depression, the individuals in this study likely have better sleep quality than the general stroke population, which does limit the generalizability of the findings of this study.

When comparing the percentage of time spent in stage N2 sleep, the control group spent $54.8 \%$ in stage N2, while the stroke participants spent $61.7 \%$ in stage N2, which is $10 \%$ more than published normative values (around 51\%). ${ }^{42}$ This is particularly interesting because stage N2 sleep is linked to consolidation of simple motor tasks. ${ }^{11}$ If spending more time in stage N2 contributes to off-line motor skill learning in individuals with chronic stroke, perhaps using sleep as a treatment method could improve functional recovery and prognosis.

There are several limitations in this pilot study. One limitation is exact location of the lesion is not known, so it 
was not possible to consider the location of the lesion in the interpretation of the data. Another limitation is the heterogeneity in age among the study participants in both groups, which limits data interpretation but improves generalizability. Furthermore, there is no consensus for reporting the time spent in the different sleep stages in percent time or in minutes. In this study, both were reported, but this lack of consensus need to be addressed to improve comparison between studies. Medication use, particularly sleep-related medications, could be a confounding factor affecting interpretation and should be considered in future studies. In addition, the conclusions should be interpreted with caution due to the low sample size and lack of correcting for multiplicity due to this being a pilot study. A larger scale study is needed to verify the findings of this pilot study.

\section{Conclusion}

In conclusion, the findings of this pilot study suggest that individuals with chronic MCA stroke have altered sleep compared with healthy controls. It appears that alterations in the sleep parameters remain even more than 6 months following a stroke, particularly changes to stage N3 sleep. However, further large-scale studies are needed to verify if less time spent in stage N3 is associated with poor motor function or if more time spent in stage N2 is associated with improved motor function and recovery in individuals with chronic stroke.

\section{Acknowledgments}

We acknowledge the sleep technicians who worked on this study. We also thank the individuals who participated in this study. This study was supported by an American Heart Association Scientist Development Grant (09SDG2060618) awarded to $\mathrm{CS}$.

\section{Disclosure}

The authors report no conflicts of interest in this work.

\section{References}

1. Pasic Z, Smajlovic D, Dostovic Z, Kojic B, Selmanovic S. Incidence and types of sleep disorders in patients with stroke. Med Arh. 2011; 65(4):225-227.

2. Johnson KG, Johnson DC. Frequency of sleep apnea in stroke and TIA patients: a meta-analysis. J Clin Sleep Med. 2010;6(2):131.

3. Lee SJ, Kim JS, Song IU, An JY, Kim YI, Lee KS. Poststroke restless legs syndrome and lesion location: anatomical considerations. Mov Disord. 2009;24(1):77-84.

4. Dobkin BH. Rehabilitation after stroke. $N$ Engl J Med. 2005;352(16): 1677-1684.

5. Yaggi HK, Concato J, Kernan WN, Lichtman JH, Brass LM, Mohsenin V. Obstructive sleep apnea as a risk factor for stroke and death. $N$ Engl $J$ Med. 2005;353(19):2034-2041.
6. Hermann DM, Bassetti CL. Sleep-related breathing and sleep-wake disturbances in ischemic stroke. Neurology. 2009;73(16):1313-1322.

7. Chan W, Coutts SB, Hanly P. Sleep apnea in patients with transient ischemic attack and minor stroke: opportunity for risk reduction of recurrent stroke? Stroke. 2010;41(12):2973-2975.

8. Siengsukon CF, Boyd LA. Sleep to learn after stroke: implicit and explicit off-line motor learning. Neurosci Lett. 2009;451(1):1-5.

9. Moser D, Anderer P, Gruber G, et al. Sleep classification according to AASM and Rechtschaffen and Kales: effects on sleep scoring parameters. Sleep. 2009;32(2):139-149.

10. Fischer S, Hallschmid M, Elsner AL, Born J. Sleep forms memory for finger skills. Proc Natl Acad Sci U S A. 2002;99(18):11987-11991.

11. Terzoudi A, Vorvolakos T, Heliopoulos I, Livaditis M, Vadikolias K, Piperidou H. Sleep architecture in stroke and relation to outcome. Eur Neurol. 2009;61(1):16-22.

12. Scheff JD, Calvano SE, Lowry SF, Androulakis IP. Modeling the influence of circadian rhythms on the acute inflammatory response. JTheor Biol. 2010;264(3):1068-1076.

13. Altemus M, Rao B, Dhabhar FS, Ding W, Granstein RD. Stress-induced changes in skin barrier function in healthy women. J Invest Dermatol. 2001;117(2):309-317.

14. Kundermann B, Krieg JC, Schreiber W, Lautenbacher S. The effect of sleep deprivation on pain. Pain Res Manag. 2004;9(1):25-32.

15. Taylor DJ, Lichstein KL, Durrence HH, Reidel BW, Bush AJ. Epidemiology of insomnia, depression, and anxiety. Sleep. 2005;28(11): 1457-1464.

16. Kyle SD, Morgan K, Espie CA. Insomnia and health-related quality of life. Sleep Med Rev. 2010;14(1):69-82.

17. Siccoli MM, Rolli-Baumeler N, Achermann P, Bassetti CL. Correlation between sleep and cognitive functions after hemispheric ischaemic stroke. Eur J Neurol. 2008;15(6):565-572.

18. Mohsenin V, Valor R. Sleep apnea in patients with hemispheric stroke. Arch Phys Med Rehabil. 1995;76(1):71-76.

19. Santamaria J, Pujol M, Orteu N, et al. Unilateral thalamic stroke does not decrease ipsilateral sleep spindles. Sleep. 2000;23(3):333-339.

20. Bassetti CL, Aldrich MS. Sleep electroencephalogram changes in acute hemispheric stroke. Sleep Med. 2001;2(3):185-194.

21. Gottselig JM, Bassetti CL, Achermann P. Power and coherence of sleep spindle frequency activity following hemispheric stroke. Brain. 2002;125(pt 2):373-383.

22. Pinto LR Jr, Silva AB, Tufik S. Rapid eye movements during paradoxical sleep in patients with cerebrovascular disease. Arq Neuropsiquiatr. 2000;58(2a):239-245.

23. Yokoyama E, Nagata K, Hirata Y, Satoh Y, Watahiki Y, Yuya H. Correlation of EEG activities between slow-wave sleep and wakefulness in patients with supra-tentorial stroke. Brain Topogr. 1996;8(3): 269-273.

24. Arzt M, Young T, Peppard PE, et al. Dissociation of obstructive sleep apnea from hypersomnolence and obesity in patients with stroke. Stroke. 2010;41(3):e129-e134.

25. Muller C, Achermann P, Bischof M, Nirkko AC, Roth C, Bassetti CL. Visual and spectral analysis of sleep EEG in acute hemispheric stroke. Eur Neurol. 2002;48(3):164-171.

26. Bliwise DL, Rye DB, Dihenia B, Gurecki P. Greater daytime sleepiness in subcortical stroke relative to Parkinson's disease and Alzheimer's disease. J Geriatr Psychiatry Neurol. 2002;15(2):61-67.

27. Giubilei $\mathrm{F}$, Iannilli $\mathrm{M}$, Vitale A, et al. Sleep patterns in acute ischemic stroke. Acta Neurol Scand. 1992;86(6):567-571.

28. Urakami Y. Relationships between sleep spindles and activities of the cerebral cortex after hemispheric stroke as determined by simultaneous EEG and MEG recordings. J Clin Neurophysiol. 2009;26(4):248-256.

29. Go AS, Mozaffarian D, Roger VL, et al; American Heart Association Statistics Committee and Stroke Statistics Subcommittee. Heart disease and stroke statistics - 2013 update: a report from the American Heart Association. Circulation. 2013;127(1):e6-e245.

30. Vock J, Achermann P, Bischof M, et al. Evolution of sleep and sleep EEG after hemispheric stroke. J Sleep Res. 2002;11(4):331-338. 
31. Leppavuori A, Pohjasvaara T, Vataja R, Kaste M, Erkinjuntti T. Insomnia in ischemic stroke patients. Cerebrovasc Dis. 2002;14(2):90-97.

32. Strine TW, Chapman DP. Associations of frequent sleep insufficiency with health-related quality of life and health behaviors. Sleep Med. 2005;6(1):23-27.

33. Agnew HW Jr, Webb WB, Williams RL. The first night effect: an EEG study of sleep. Psychophysiology. 1966;2(3):263-266.

34. Rechtschaffen A, Dement W. Studies on the relation of narcolepsy, cataplexy, and sleep with low voltage random EEG activity. Res Publ Assoc Res Nerv Ment Dis. 1967;45:488-505.

35. Pivik RT, Broughton RJ, Coppola R, Davidson RJ, Fox N, Nuwer MR. Guidelines for the recording and quantitative analysis of electroencephalographic activity in research contexts. Psychophysiology. 1993;30(6):547-558.

36. Berry RB, Brooks R, Gamaldo CE, et al. The AASM Manual for the Scoring of Sleep and Associated Events: Rules, Terminology and Technical Specifications, Version 2.1. Darien, Illinois: American Academy of Sleep Medicine; 2014

37. Lai S-M, Duncan PW, Keighley J. Prediction of functional outcome after stroke comparison of the Orpington Prognostic Scale and the NIH Stroke Scale. Stroke. 1998;29(9):1838-1842.
38. Nudo RJ. Mechanisms for recovery of motor function following cortical damage. Curr Opin Neurobiol. 2006;16(6):638-644.

39. Poryazova R, Huber R, Khatami R, et al. Topographic sleep EEG changes in the acute and chronic stage of hemispheric stroke. J Sleep Res. 2015;24(1):54-65.

40. Huber R, Ghilardi MF, Massimini M, Tononi G. Local sleep and learning. Nature. 2004;430(6995):78-81.

41. Huber R, Ghilardi MF, Massimini M, et al. Arm immobilization causes cortical plastic changes and locally decreases sleep slow wave activity. Nat Neurosci. 2006;9(9):1169-1176.

42. Danker-Hopfe H, Schäfer M, Dorn H, et al. Percentile reference charts for selected sleep parameters for 20-to 80 -year-old healthy subjects from the SIESTA database. Somnologie. 2005;9(1):3-14.

43. Buysse DJ, Reynolds CF 3rd, Monk TH, Berman SR, Kupfer DJ The Pittsburgh Sleep Quality Index: a new instrument for psychiatric practice and research. Psychiatry Res. 1989;28(2):193-213.
Nature and Science of Sleep

\section{Publish your work in this journal}

Nature and Science of Sleep is an international, peer-reviewed, open access journal covering all aspects of sleep science and sleep medicine, including the neurophysiology and functions of sleep, the genetics of sleep, sleep and society, biological rhythms, dreaming, sleep disorders and therapy, and strategies to optimize healthy sleep. The journal welcomes

\section{Dovepress}

original research, clinical \& epidemiological studies, reviews \& evaluations, case reports and extended reports. The manuscript management system is completely online and includes a very quick and fair peerreview system, which is all easy to use. Visit http://www.dovepress.com/ testimonials.php to read real quotes from published authors. 